\title{
Vibrations of a simply supported beam with a fractional viscoelastic material model - supports movement excitation
}

\author{
Jan Freundlich \\ Faculty of Automotive and Construction Machinery Engineering, Warsaw University of Technology, Narbutta 84, \\ 02-524 Warsaw, Poland \\ Tel.: +48 22 2348459; Fax: +4822 2348286; E-mail: jfr@simr.pw.edu.pl
}

\begin{abstract}
The paper presents vibration analysis of a simply supported beam with a fractional order viscoelastic material model. The Bernoulli-Euler beam model is considered. The beam is excited by the supports movement. The Riemann - Liouville fractional derivative of order $0<\alpha \leqslant 1$ is applied. In the first stage, the steady-state vibrations of the beam are analyzed and therefore the Riemann - Liouville fractional derivative with lower terminal at $-\infty$ is assumed. This assumption simplifies solution of the fractional differential equations and enables us to directly obtain amplitude-frequency characteristics of the examined system. The characteristics are obtained for various values of fractional derivative of order $\alpha$ and values of the Voigt material model parameters. The studies show that the selection of appropriate damping coefficients and fractional derivative order of damping model enables us to fit more accurately dynamic characteristic of the beam in comparison with using integer order derivative damping model.
\end{abstract}

Keywords: Fractional viscoelasticity, beam vibration, damping of vibrations

\section{Introduction}

Fractional derivatives are applied in many fields, such as control systems, bioengineering, viscoelasticity, heat transfer, electro-chemistry, dynamics, economics etc [17,22]. The survey of the documents and events in the area of fractional calculus can be found in the paper by Machado, Kiryakova and Mainardi [17] and review focused on the application of fractional calculus in dynamic problems of solids is presented in paper by Rossikhin and Shitikova [22]. Fractional derivatives, in contrast to integer order derivatives, where the derivative depends only on the local condition of the function, is not local and depends on "history" of the function [7,8]. This property is exploited in processes which depend on the time history of the input (hereditary processes). In particular, the damping properties of viscoelastic material can be modeled using the fractional order derivative and enables us to use smaller number of parameters than using integer order derivatives. The fractional models more accurately describe damping properties of a variety of materials over wide range of frequencies [2,4,5]. The fractional model of viscoelasticity may be derived from integer order description using Boltzmann superposition principle e.g. [2, 7]. Among different definitions of the fractional derivative, the ones provided by Riemann-Liouville and Caputo are commonly used in various engineering applications including viscoelastic damping models $[6,18,22]$. With some minimal restrictions, the two definitions produce completely equivalent mathematical models of the linear viscoelastic phenomenon [5].

The general linear integer order of viscoelasticity is described by the following relationship [18]:

$$
\sum_{k=0}^{n} a_{k} \frac{d^{k} \sigma(t)}{d t^{k}}=\sum_{k=0}^{m} b_{k} \frac{d^{k} \varepsilon(t)}{d t^{k}} \quad m, n=0,1,2,3 \ldots
$$


Use of large number of terms in formula (1) leads to complicated differential equations which are difficult to solve. More efficient solution which preserves the linearity of the model is application of the fractional viscoelastic model $[7,8]$. The fractional model can be expressed as

$$
\sum_{k=0}^{n} a_{k} D^{\alpha_{k}} \sigma(t)=\sum_{k=0}^{m} b_{k} D^{\beta_{k}} \varepsilon(t)
$$

Adolfsson et al. [2] shown that complicated multi-parameter models converge to fractional models with one single parameter. Rayleigh (proportional) damping is commonly used in simulating dynamic response of structures, especially when continuous systems are modeled by the finite element technique $[1,9,15,25]$. In this model, the damping matrix $\mathrm{C}$ is defined as

$$
\mathbf{C}=\alpha \mathbf{M}+\beta \mathbf{K}
$$

where $\boldsymbol{M}$ and $\boldsymbol{K}$ are the mass and stiffness matrices respectively, and $\alpha$ and $\beta$ are constants. Rayleigh damping and the generalized Maxwell model are equivalent for small to moderate values of damping ratio [23]. In the case when $\alpha=0$, the term proportional to stiffness matrix $\mathrm{K}$ represents Voigt material model [15]. Namely, the Voigt viscoelastic material model is useful for the description of the material internal damping and is commonly used in modeling internal damping in structural elements $[1,7,14,16,18]$. Unfortunately this model is not accurate for describing a wide class of nearly elastic engineering materials and the resulting loss factor is proportional to the excitation frequency [13]. It also overestimates damping for higher vibration frequencies $[1,9,15]$. Thus, the fractional viscoelastic Voigt material model could more accurately describe material damping properties for wider range of frequencies $[6,12,15,18]$. This fractional model is defined by the following equation:

$$
\sigma=\left(E \varepsilon+E_{t \alpha} \frac{d^{\alpha} \varepsilon(t)}{d t}\right)=E\left(\varepsilon+\mu_{\alpha} \frac{d^{\alpha} \varepsilon(t)}{d t}\right)
$$

where

$\mu_{\alpha}-E_{t \alpha} / E$,

$E$ - Young modulus of the beam material,

$E_{t \alpha}$ - damping coefficient.

The fractional derivative of order $\alpha$ is defined as below:

$$
\frac{d^{\alpha}}{d t} f(t) \equiv{ }_{a} D_{t}^{\alpha} f(t) \equiv \frac{1}{\Gamma(1-\alpha)} \frac{d}{d t} \int_{a}^{t} \frac{f(\tau) d \tau}{(t-\tau)^{\alpha+1-m}}
$$

and the fractional derivative order $\alpha$ for many real materials is $0<\alpha \leqslant 1[8,11,13,19]$ while the lower limit of the integral $a=0$.

Numerous research works related to phenomena described by fractional models present solutions of transient responses, e.g. [3,16,21,22]. In general, the solutions are often expressed as series expansion $[4,8,10]$, which are not very useful for practical applications because of its slow convergence rate [24]. The steady-state responses of mechanical structures are frequently investigated engineering problems, for example in order to obtain the amplitudefrequency response of the examined system.

Thus, the aim of this study is to show that the fractional viscoelastic Voigt material model allows for better description of the damping properties in the larger range of frequency then in the case of the use of a similar integer order model. In the engineering practice, beams are frequently used for modeling dynamic of complex structures e.g. $[3,4,14,16]$. The number of publications considering steady-state beam vibrations with fractional viscoelstic model is limited [22] and therefore the steady-state vibration of a simply supported beam excited by support movement is considered to show advantage of using the fractional material Voigt model in comparison to the integer model.

An engineering approach demonstrated by Rossikhin and Shitikova [21] is utilized. This approach simplifies the solution and makes it more useful for the engineering application. Namely, this approach neglects the drift of the 


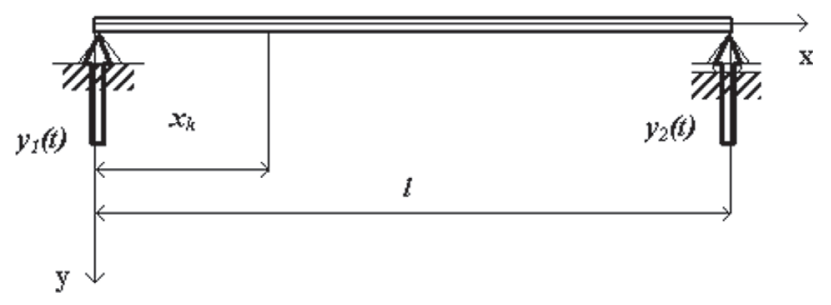

Fig. 1. Schema of the analyzed system.

equilibrium position of the oscillator. The quoted assumption allows for use of the lower limit a of the integral in Eq. (5) in minus infinity, therefore the used fractional derivative has the form

$$
\frac{d^{\alpha}}{d t} f(t) \equiv \dot{f}^{\alpha}(t) \equiv{ }_{-\infty} D_{t}^{\alpha} f(t) \equiv \frac{1}{\Gamma(1-\alpha)} \frac{d}{d t} \int_{-\infty}^{t} \frac{f(\tau) d \tau}{(t-\tau)^{\alpha}}
$$

Additionally, the assumption of the lower limit of the integral in a minus infinity allows for straightforward calculations of the fractional derivatives $[10,20,21]$ i.e.

$$
\frac{d^{\alpha}}{d t} \sin (\nu t)=\nu^{\alpha} \sin \left(\nu t+\frac{\pi \alpha}{2}\right)
$$

The last relationship simplifies obtaining the solution.

\section{Governing equations}

The equation of motion of the examined beam is derived under the assumption of the Bernoulli-Euler theory, neglecting rotary inertia and shear deformation. The uniform bending stiffness and mass density are assumed as well. Using these assumptions, the governing equation of forced transverse motion of the beam is obtained as shown

$$
E J\left(\frac{\partial^{4} y(x, t)}{\partial x^{4}}+\mu_{\alpha} \frac{d^{\alpha}}{d t}\left(\frac{\partial^{4} y(x, t)}{\partial x^{4}}\right)\right)+A \rho \frac{\partial^{2} y(x, t)}{\partial t^{2}}=p(x, t)
$$

$A$ - cross-section area of the beam,

$J$ - moment of inertia of the beam cross-section,

$\rho$ - material mass density of the beam,

$y(x, t)-$ transversal displacement of the neutral beam axis (Fig. 1),

$t$ - time,

$x$ - longitudinal coordinate,

$p(x, t)$ - external transverse load.

In the present research, only the kinematic excitation is analyzed and therefore it is assumed that the transverse load $p(x, t)$ is equal to zero. Without loss of generality, a simply-supported beam excited by the support movement is analyzed (Fig. 1). A similar model of the beam was utilized by Freundlich and Pietrzakowski for an investigation of the behavior of piezoelectric sensors which were used for measurement of the vibrations of a beam [14]. The boundary conditions for the analysed beam are assumed as follows [14]

$$
\begin{aligned}
& y(0, t)=y_{1}(t), \quad y(l, t)=y_{2}(t) \\
& \left.\frac{\partial^{2} y}{\partial x^{2}}\right|_{x=0}=\left.\frac{\partial^{2} y}{\partial x^{2}}\right|_{x=l}=0
\end{aligned}
$$

Introducing the geometrical relation of the supports movement, the transversal displacement of the neutral beam axis can be expressed as

$$
y(x, t)=\left(1-\frac{x}{l}\right) y_{1}(t)+\frac{x}{l} y_{2}(t)+w(x, t)
$$


Substituting Eqs (9)-(11) into the governing Eq. (8) the equation describing the relative movement of the beam is obtained

$$
\frac{\partial^{4} w(x, t)}{\partial x^{4}}+\eta \frac{d^{\alpha}}{d t}\left(\frac{\partial^{4} w(x, t)}{\partial x^{4}}\right)+a^{2} \frac{\partial^{2} w(x, t)}{\partial t^{2}}=-a^{2}\left(\left(1-\frac{x}{l}\right) \ddot{y}_{1}(t)+\frac{x}{l} \ddot{y}_{2}(t)\right)
$$

where

$$
a^{2}=\frac{\rho A}{E J}, \quad \eta=\frac{\mu_{\alpha}}{E J}
$$

In the first step, the general solution of Eq. (12) is obtained by the separation of variables method [25]

$$
\frac{\partial^{4} w(x, t)}{\partial x^{4}}+\eta \frac{d^{\alpha}}{d t}\left(\frac{\partial^{4} w(x, t)}{\partial x^{4}}\right)+a^{2} \frac{\partial^{2} w(x, t)}{\partial t^{2}}=0
$$

The solution of Eq. (13) is assumed as

$$
w(x, t)=W(x) T(t)
$$

The fractional derivative can be computed as follows

$$
\frac{d^{\alpha}}{d t}\left(\frac{\partial^{4} w(x, t)}{\partial x^{4}}\right)=W^{I V}(x) \dot{T}^{\alpha}(t)
$$

Substituting Eqs (14) and (15) into Eq. (16) the following equation is obtained

$$
W^{I V}(x)\left[T(t)+\eta \dot{T}^{\alpha}(t)\right]+a^{2} W(x) \ddot{T}(t)=0
$$

Grouping the terms with variables $x$ and $t$ separately, and equating to the same constant $k$

$$
\frac{W^{I V}(x)}{W(x)}=-\frac{a^{2} \ddot{T}(t)}{T(t)+\eta \dot{T}^{\alpha}(t)}=k^{4}
$$

the system of two equations is obtained

$$
\begin{aligned}
& W^{I V}(x)-k^{4} W(x)=0 \\
& \ddot{T}(t)+\frac{k^{4}}{a^{2}} \eta \dot{T}^{\alpha}(t)+\frac{k^{4}}{a^{2}} T(t)=0
\end{aligned}
$$

The solution of Eq. (18) is well-known e.g. [22,25] and the normalized eigenfunctions for a simply supported beam are expressed as

$$
W_{n}(x)=\sin \frac{n \pi x}{l} \quad n=1,2,3 \ldots
$$

where

$l$ - length of the beam.

The eigenfunctions are employed to find steady-state response of the beam which is excited by the supports movement. Their movement is assumed as

$$
y_{1}(0, t)=y_{10} \sin (\nu t), \quad y_{2}(l, t)=y_{20} \sin (\nu t+\phi)
$$

where

$y_{01}, y_{02}-$ the displacement amplitudes at the beam edges, $\phi$ - phase angle, 
$\nu$ - excitation frequency.

Substituting expressions Eq. (21) into Eq. (12) the following equation is obtained

$$
\begin{aligned}
& \frac{\partial^{4} w(x, t)}{\partial x^{4}}+\eta \frac{d^{\alpha}}{d t}\left(\frac{\partial^{4} w(x, t)}{\partial x^{4}}\right)+a^{2} \frac{\partial^{2} w(x, t)}{\partial t^{2}} \\
= & -a^{2}\left(\left(1-\frac{x}{l}\right)\left(-\nu^{2} y_{10} \sin (\nu t)\right)+\frac{x}{l}\left(-\nu^{2} y_{20} \sin (\nu t+\phi)\right)\right)
\end{aligned}
$$

The steady state response of the beam is assumed as

$$
w(x, t)=\sum_{n=1}^{\infty} \xi_{n}(t) W_{n}(x)
$$

The corresponding derivatives are evaluated bellow

$$
\begin{aligned}
\frac{\partial^{4} w(x, t)}{\partial x^{4}} & =W_{n}^{I V}(x) \xi_{n}(t) \\
\frac{d^{\alpha}}{d t}\left(\frac{\partial^{4} w(x, t)}{\partial x^{4}}\right) & =W_{n}^{I V} \dot{\xi}_{n}^{\alpha}(t) \\
\frac{\partial^{2} w(x, t)}{\partial t^{2}} & =W_{n} \ddot{\xi}_{n}(t)
\end{aligned}
$$

Substituting Eqs (24)-(26) into Eq. (22) we get

$$
\sum_{n=1}^{\infty}\left[W_{n}^{I V} \xi_{n}(t)+\eta W_{n}^{I V} \dot{\xi}_{n}^{\alpha}(t)+a^{2} W \ddot{\xi}_{n}(t)\right]=q(x, t)
$$

where $q(x, t)$ denotes RHS of Eq. (22).

Taking into consideration that

$$
W_{n}^{I V}(x)=W_{n}(x) k_{n}^{4}
$$

Substituting Eq. (28) into Eq. (27) the following expression is obtained

$$
\sum_{n=1}^{\infty}\left[k_{n}^{4} W_{n}(x) \xi_{n}(t)+\eta k_{n}^{4} W_{n} \dot{\xi}_{n}^{\alpha}(t)+a^{2} W \ddot{\xi}_{n}(t)\right]=q(x, t)
$$

Denoting $k^{4}=\omega^{2} a^{2}$ in Eq. (29) results in following equation:

$$
\sum_{n=1}^{\infty}\left[\omega_{n}^{2} W_{n}(x) \xi_{n}(t)+\eta \omega_{n}^{2} W_{n} \dot{\xi}_{n}^{\alpha}(t)+W_{n}(x) \ddot{\xi}_{n}(t)\right]=\frac{q(x, t)}{a^{2}}
$$

Expressing $q(x, t)$ by the series expansion

$$
q(x, t)=\sum_{n=1}^{\infty} Q_{n}(t) W_{n}(x)=\sum_{n=1}^{\infty} Q_{n}(t) \sin \frac{n \pi x}{l}
$$

integrating over $x=[0, l]$ and using the orthogonality property of eigenfunctions, the $\mathrm{Q}_{n}$ coefficients can be expressed as

$$
Q_{n}=\frac{1}{\gamma^{2}} \int_{0}^{l} q(x, t) W_{n}(x) d x
$$


where $\gamma^{2}=\int_{0}^{l} W_{n}^{2}(x) d x$.

After simplification the following formula is obtained:

$$
\sum_{n=1}^{\infty}\left[\omega_{n}^{2} \xi_{n}(t)+\eta \omega_{n}^{2} \dot{\xi}_{n}^{\alpha}(t)+\ddot{\xi}_{n}(t)-\frac{1}{a^{2}} \sum_{n=1}^{\infty} Q_{n}(t)\right] \cdot W_{n}(x)=0
$$

This equation is satisfied when the expression in brackets is equal to zero i.e.

$$
\ddot{\xi}_{n}(t)+\eta \omega_{n}^{2} \dot{\xi}_{n}^{\alpha}(t)+\omega_{n}^{2} \xi_{n}(t)=\frac{Q_{n}(t)}{a^{2}}
$$

For the simply supported beam

$$
\gamma^{2}=\int_{0}^{l} \sin ^{2}\left(\frac{n \pi x}{l}\right) d x=\frac{l}{2}
$$

and then

$$
Q_{n}(t)=\frac{2 a^{2}}{l} \cdot \int_{0}^{l}\left[\nu^{2} y_{10} \sin (\nu t)\left(1-\frac{x}{l}\right)+\nu^{2} y_{20} \sin (\nu t+\phi) \frac{x}{l}\right] \sin \frac{n \pi x}{l} d x
$$

Evaluating the integral in Eq. (36) the terms $Q_{n}$ are obtained

$$
Q_{n}(t)=\frac{2 a^{2} \nu^{2}}{n \pi}\left\{y_{10} \sin \nu t-y_{20}(-1)^{n} \sin (\nu t+\phi)\right\}
$$

Substituting Eq. (37) in Eq. (34) we obtain

$$
\ddot{\xi}_{n}(t)+\eta \omega_{n}^{2} \dot{\xi}_{n}^{\alpha}(t)+\omega_{n}^{2} \xi_{n}(t)=\frac{2 \nu^{2}}{n \pi}\left\{y_{10} \sin \nu t-y_{20}(-1)^{n} \sin (\nu t+\phi)\right\}
$$

Then, using trigonometric identities and doing some mathematical transformations we get a system of equations

$$
\ddot{\xi}_{n}(t)+\eta \omega_{n}^{2} \dot{\xi}_{n}(t)+\omega_{n}^{2} \xi_{n}(t)=L_{n} \sin \nu t+M_{n} \cos \nu t
$$

where

$$
L_{n}=\frac{2 \nu^{2}}{n \pi}\left\{y_{10}-y_{20}(-1)^{n} \cos \phi\right\} \text { and } M_{n}=-\frac{2 \nu^{2} y_{20}(-1)^{n} \sin \phi}{n \pi}
$$

The solution of each $n$-th equation is assumed as

$$
\xi_{n}(t)=A_{n} \sin \left(\nu t+\delta_{n}\right)
$$

The corresponding derivatives are as follows

$$
\dot{\xi}_{n}^{\alpha}(t)=A_{n} \nu^{\alpha} \sin \left(\nu t+\frac{\pi \cdot \alpha}{2}+\delta_{n}\right), \quad \ddot{\xi}_{n}(t)=-A_{n} \nu^{2} \sin \left(\nu t+\delta_{n}\right)
$$

Substitution of Eqs (40) and (41) into Eq. (39) yields the equation

$$
\begin{aligned}
& {\left[A_{n}\left(\omega_{n}^{2}-\nu^{2}\right)+A_{n} \eta \nu^{\alpha} \omega_{n}^{2} \cos \frac{\pi \cdot \alpha}{2}\right] \sin \left(\nu t+\delta_{n}\right)+\left(A_{n} \eta \nu^{\alpha} \omega_{n}^{2} \sin \frac{\pi \cdot \alpha}{2}\right) \cos \left(\nu t+\delta_{n}\right)} \\
& =\sin \left(\nu t+\delta_{n}\right)\left[L_{n} \cos \delta_{n}+M_{n} \sin \delta_{n}\right]+\cos \left(\nu t+\delta_{n}\right)\left[M_{n} \cos \delta_{n}-L_{n} \sin \delta_{n}\right]
\end{aligned}
$$


Amplitudes $A_{n}$ and the phase angles $\delta_{n}$ can be calculated by equating the coefficients of the sine and cosine of the right and left sides of the Eq. (42). Then, the steady-state response of the beam has the form

$$
w(x, t)=\sum_{n=1}^{\infty} A_{n} \sin \left(\nu t+\delta_{n}\right) \sin \frac{n \pi x}{l}
$$

Where amplitudes $A_{n}$ are

$$
A_{n}=\sqrt{\frac{\left(L_{n}^{2}+M_{n}^{2}\right)}{\left(\omega_{n}^{2}-\nu^{2}+\eta \nu^{\alpha} \omega_{n}^{2} \cos \frac{\pi \cdot \alpha}{2}\right)^{2}+\left(\eta \nu^{\alpha} \omega_{n}^{2} \sin \frac{\pi \cdot \alpha}{2}\right)^{2}}}
$$

and the phase angles $\delta_{n}$ is computed from equation

$$
\operatorname{tg} \delta_{n}=\frac{\left(\omega_{n}^{2}-\nu^{2}+A_{n} \eta \nu^{\alpha} \omega_{n}^{2} \cos \frac{\pi \cdot \alpha}{2}\right) M_{n}-\eta \nu^{\alpha} \omega_{n}^{2} \sin \frac{\pi \cdot \alpha}{2} L_{n}}{\left(\omega_{n}^{2}-\nu^{2}+A_{n} \eta \nu^{\alpha} \omega_{n}^{2} \cos \frac{\pi \cdot \alpha}{2}\right) L_{n}+\eta \nu^{\alpha} \omega_{n}^{2} \sin \frac{\pi \cdot \alpha}{2} M_{n}}
$$

The variation of vibration amplitudes with the excitation frequency and longitudinal coordinate is given by

$$
A(x, \nu)=\sqrt{\left(\sum_{n=1}^{\infty} A_{n}^{2} \cos \delta_{n}\right)^{2}+\left(\sum_{n=1}^{\infty} A_{n}^{2} \sin \delta_{n}\right)^{2}} \sin \frac{n \pi x}{l}
$$

where

$$
\cos \delta_{n}=\frac{\left(\omega_{n}^{2}-\nu^{2}+A_{n} \eta \nu^{\alpha} \omega_{n}^{2} \cos \frac{\pi \cdot \alpha}{2}\right) L_{n}+\eta \nu^{\alpha} \omega_{n}^{2} \sin \frac{\pi \cdot \alpha}{2} M_{n}}{\sqrt{\left[\left(\omega_{n}^{2}-\nu^{2}+\eta \nu^{\alpha} \omega_{n}^{2} \cos \frac{\pi \cdot \alpha}{2}\right)^{2}+\left(\eta \nu^{\alpha} \omega_{n}^{2} \sin \frac{\pi \cdot \alpha}{2}\right)^{2}\right]\left(L_{n}^{2}+M_{n}^{2}\right)}}
$$

and

$$
\sin \delta_{n}=\frac{\left(\omega_{n}^{2}-\nu^{2}+A_{n} \eta \nu^{\alpha} \omega_{n}^{2} \cos \frac{\pi \cdot \alpha}{2}\right) M_{n}-\eta \nu^{\alpha} \omega_{n}^{2} \sin \frac{\pi \cdot \alpha}{2} L_{n}}{\sqrt{\left[\left(\omega_{n}^{2}-\nu^{2}+\eta \nu^{\alpha} \omega_{n}^{2} \cos \frac{\pi \cdot \alpha}{2}\right)^{2}+\left(\eta \nu^{\alpha} \omega_{n}^{2} \sin \frac{\pi \cdot \alpha}{2}\right)^{2}\right]\left(L_{n}^{2}+M_{n}^{2}\right)}}
$$

\section{Influence of the fractional derivative order on amplitude-frequency characteristics}

In the first stage, the achieved formula (46) is applied to examine the influence of fractional derivative order $\alpha$ and the damping coefficient $\eta$ on the amplitude-frequency relationships of the beam.

The calculations are performed for several values of fractional derivative order $\alpha$, phase angle $\varphi=\pi / 6$ and damping coefficients $\eta=2 \cdot 10^{-5}$ and $\eta=5 \cdot 10^{-5}\left[\mathrm{~s}^{\alpha} /\left(\mathrm{N} \cdot \mathrm{m}^{2}\right)\right]$.

The examined beam is 3 meters in length, with the I-shaped cross-section. The dimensions of the cross-section are $60 \mathrm{~mm}$ in width, $120 \mathrm{~mm}$ in height, $6 \mathrm{~mm}$ in thickness of edges and $3 \mathrm{~mm}$ with thickness of the wall. Vibration amplitudes of the beam are calculated for a point located at $x_{k}=0.9 \mathrm{~m}$ from the left edge of the beam (Fig. 1).

As previously mentioned, the Voigt integer order model overestimates damping for higher vibration frequencies and causes diminishing resonant amplitude values that correspond to higher vibration modes. It is visible for resonance curves achieved for derivative order $\alpha=1$ (Figs 2 and 6). 


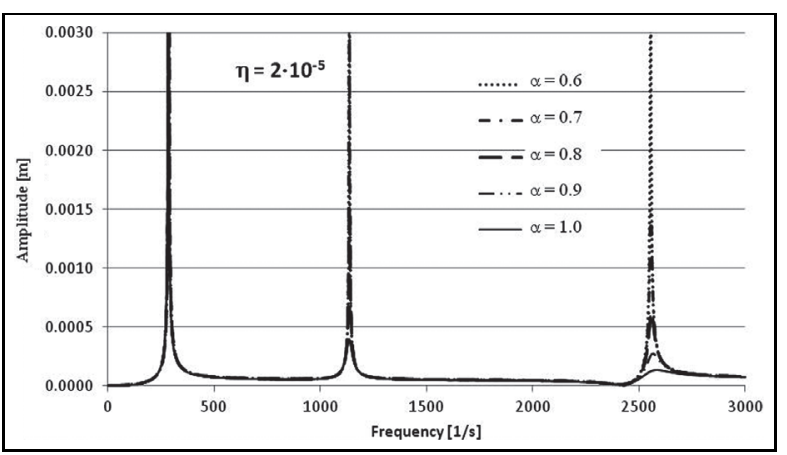

Fig. 2. Amplitude-frequency characteristic $\eta=2 \cdot 10^{-5}$.

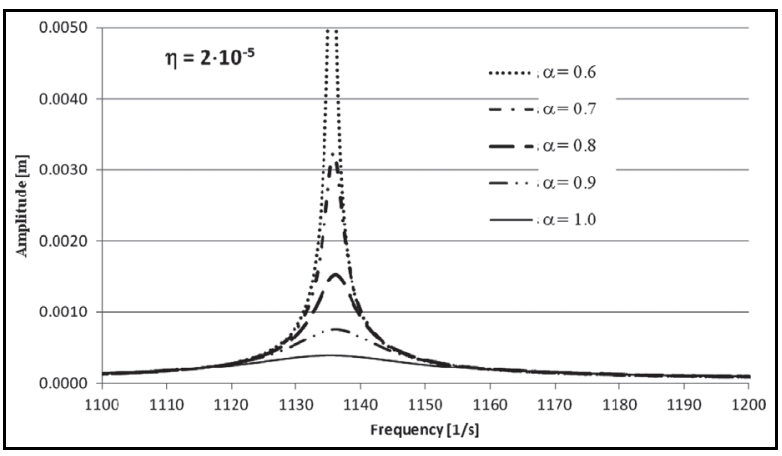

Fig. 4. Amplitude-frequency characteristic - 2-nd resonance.

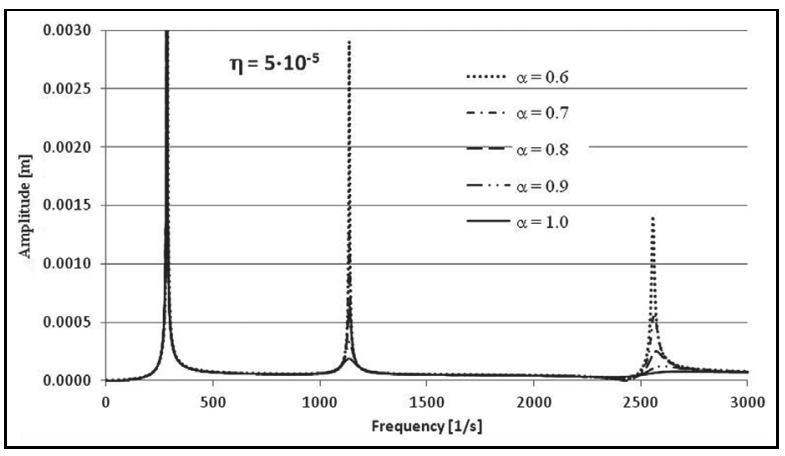

Fig. 6. Amplitude-frequency characteristic $\eta=5 \cdot 10^{-5}$

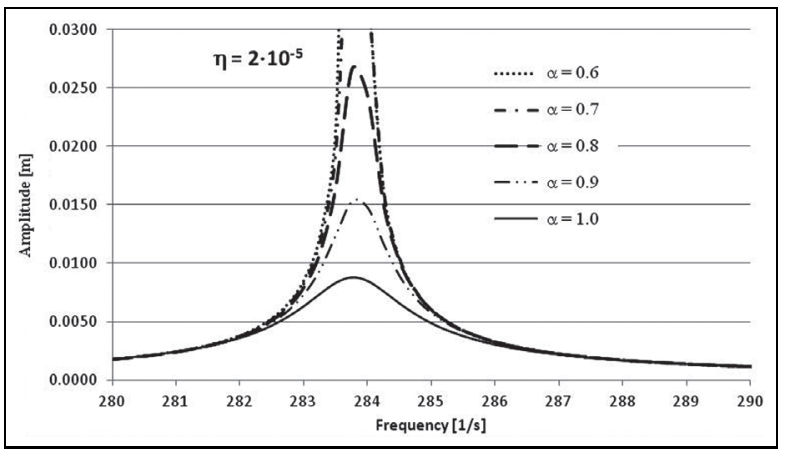

Fig. 3. Amplitude-frequency characteristic - 1-st resonance.

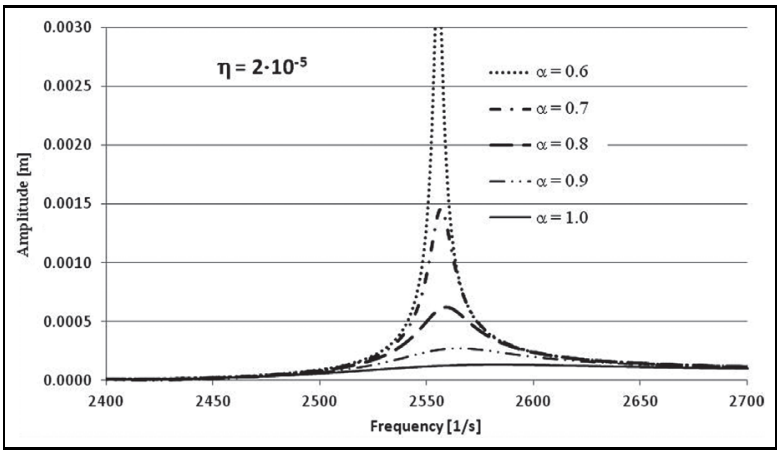

Fig. 5. Amplitude-frequency characteristic -3-rd resonance.

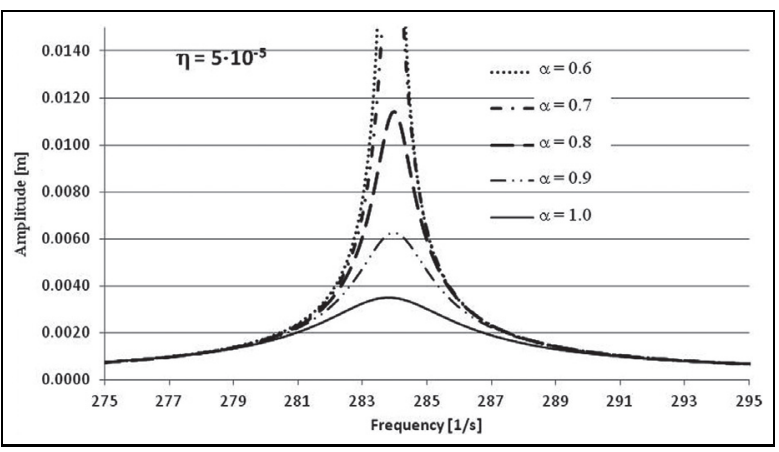

Fig. 7. Amplitude-frequency characteristic - 1-st resonance.

The achieved amplitude-frequency relationship for damping coefficient $\eta=2 \cdot 10^{-5}\left[\mathrm{~s}^{\alpha} /\left(\mathrm{N} \cdot \mathrm{m}^{2}\right)\right]$ is shown in Figs 2 to 5. It is visible that decreasing fractional derivative order causes increase in vibration amplitudes, especially for higher frequencies. It is more noticeable in Figs 3-5 where enlarged graphs are shown. Larger amplitudes for the higher excitation frequencies are more evident for the damping coefficient $\eta=5 \cdot 10^{-5}\left[\mathrm{~s}^{\alpha} /\left(\mathrm{N} \cdot \mathrm{m}^{2}\right)\right]$. The corresponding results are shown in Figs 6 to 9. The peaks for the second and third resonance practically disappeared in the case of the derivative order $\alpha=1$ (Figs 2 and 6) whereas for the order $\alpha \leqslant 0.8$ these peaks are noticeable.

In the next stage, the selection possibility of the parameters of the fractional viscoelastic Voigt model is presented. These parameters are selected in a way that the resonance curve obtained using the fractional viscoelastic Voigt model is similar to the curve obtained using the integer model and in the area of the first resonant frequency the curves are almost identical. As an example, the calculations of two resonance curves are performed. The calculations are carried out for integer order Voigt model $(\alpha=1)$ and damping coefficient $\eta=5.9 \cdot 10^{-5}\left[\mathrm{~s} /\left(\mathrm{N} \cdot \mathrm{m}^{2}\right)\right]$ and for the 


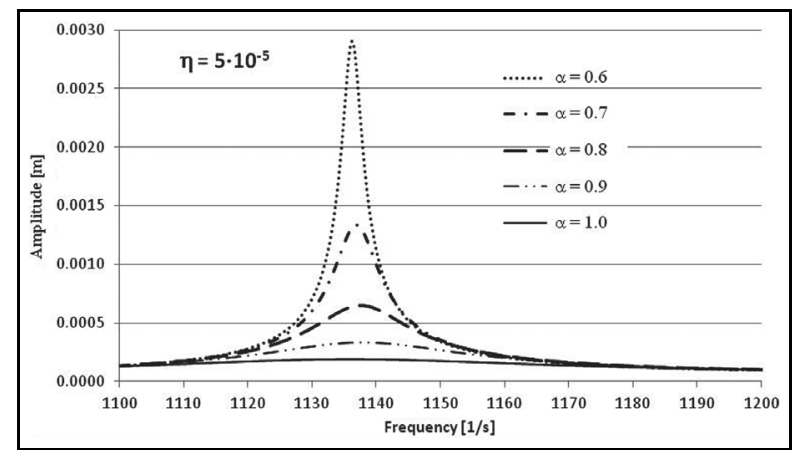

Fig. 8. Amplitude-frequency characteristic - 2-nd resonance.

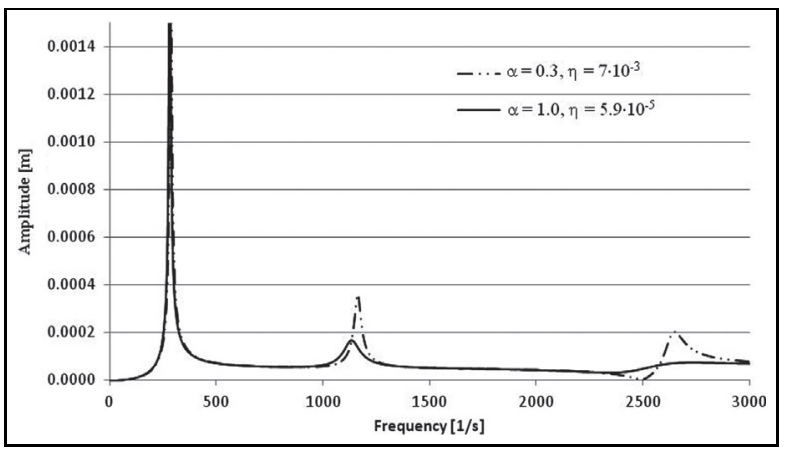

Fig. 10. Comparison of amplitude-frequency characteristics for $\alpha=$ $0.3 \eta=7 \cdot 10^{-3}$ and $\alpha=1.0 \eta=5.9 \cdot 10^{-3}$.

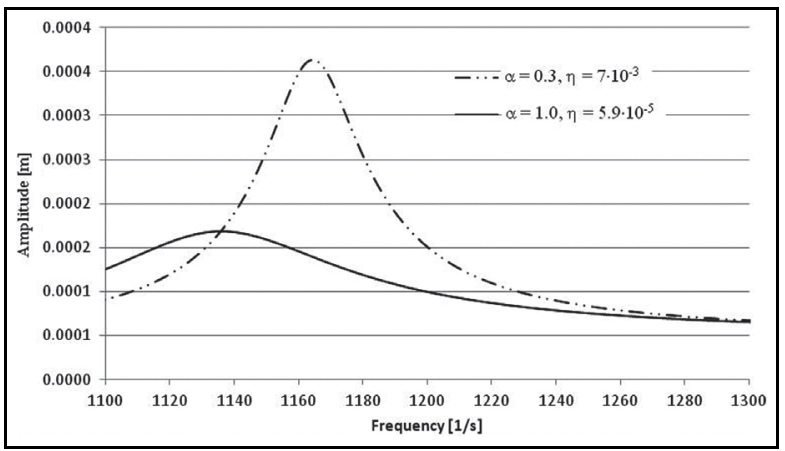

Fig. 12. Comparison of amplitude-frequency characteristics for $\alpha=$ $0.3 \eta=7 \cdot 10^{-3}$ and $\alpha=1.0 \eta=5.9 \cdot 10^{-3}-2$-nd resonance.

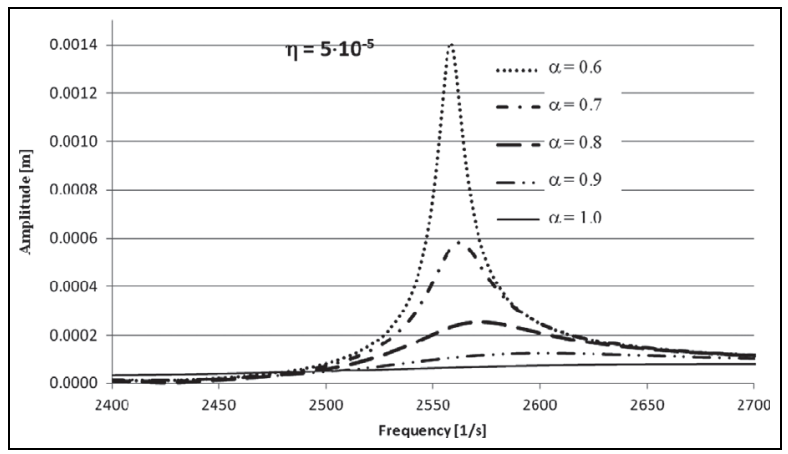

Fig. 9. Amplitude-frequency characteristic - 3-th resonance.

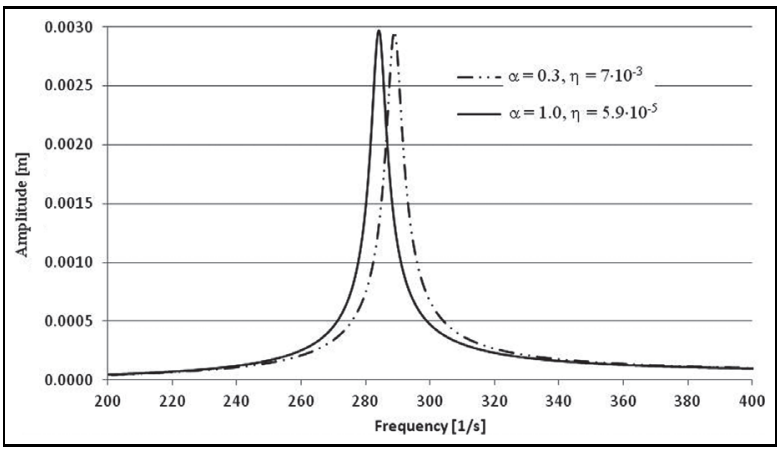

Fig. 11. Comparison of amplitude-frequency characteristics for $\alpha=$ $0.3 \eta=7 \cdot 10^{-3}$ and $\alpha=1.0 \eta=5.9 \cdot 10^{-3}-1$-st resonance.

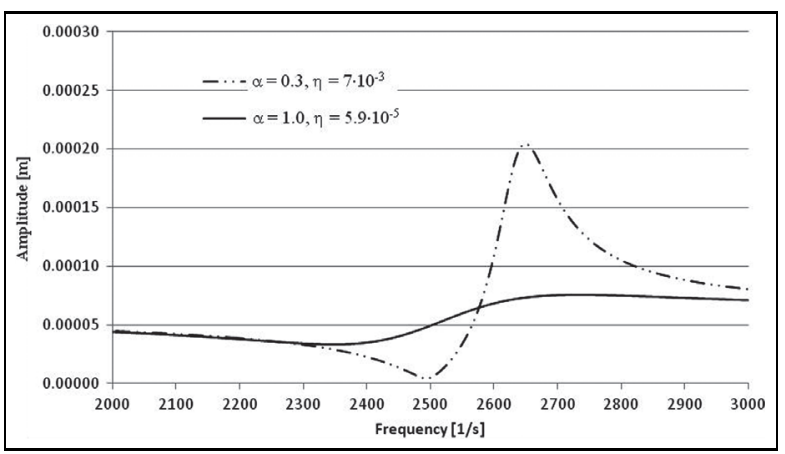

Fig. 13. Comparison of amplitude-frequency characteristics for $\alpha=$ $0.3 \eta=7 \cdot 10^{-3}$ and $\alpha=1.0 \eta=5.9 \cdot 10^{-3}-3$-th resonance.

fractional model, where $\alpha=0.3$ and $\eta=5.9 \cdot 10^{-5}\left[\mathrm{~s}^{\alpha} /\left(\mathrm{N} \cdot \mathrm{m}^{2}\right)\right]$. The obtained resonance curves are shown in Fig. 10 $\div$ 13. It is visible that that the curves are almost identical in the first natural frequency area (Figs 10 and 11 ). In the case of fractional order Voigt model the resonant amplitudes corresponding to the second and third resonant frequencies are more noticeable than in the case of the integer order derivative. In case of the second resonant frequency, the amplitude calculated using fractional model is approximately two times greater than for the integer model (Figs 10 and 12).

\section{Conclusions}

- The steady-state solution of a simply-supported Bernoulli-Euler beam with fractional Voigt material model 
excited by support movement is achieved.

- The method of solution is similar to that used for the integer order Voigt material model and could be useful for engineering applications.

- The fractional viscoelastic model allows for better description of the damping properties in the larger range of frequency then in the case of the integer order model, i.e. using fewer number of terms in Eq. (1).

- The selection of fractional Voigt model parameters in order to obtain appropriate resonance curve is presented. The selection preserve similar shape resonance curves in the case of fractional and integer order Voigt material model is shown. The fractional viscoelastic model, in contrary to integer model, produces larger vibration amplitudes for higher resonant frequencies.

- Experimental studies should be undertaken to verify the calculated amplitude-frequency characteristics.

\section{References}

[1] Abaqus, Online Documentation: Version 6.11-1, Dassault Systèmes, 2011.

[2] K. Adolfsson, M. Enelund and P. Olsson, On the fractional order model of viscoelasticity, Mechanics of Time-Dependent Materials 9 (2005), 15-34.

[3] O.P. Agrawal, Analytical solution for stochastic response of a fractionally damped beam, ASME Journal of Vibration and Acoustics 126 (Oct 2004), 561-566.

[4] T.M. Atanackovic and B. Stankovic, Dynamics of a viscoelastic rod of fractional derivative type, ZAMM_Z Angew Math Mech 82(6) (2002), 377-386.

[5] R. Bagley, On the equivalence of the Riemann-Liouville and the Caputo fractional order derivatives in modeling of linear viscoelastic materials, Fractional Calculus and Applied Analysis 10(2) (2010).

[6] R.L. Bagley and P.J. Torvik, Fractional calculus - A different approach to the analysis of viscoelastically damped structures, AIAA Journal 21(5) (1983), 741-748.

[7] R.L. Bagley and P.J. Torvik, A theoretical basis for the application of fractional calculus to viscoelasticity, Journal of Rheology 27(3) (1983), 201-210.

[8] R.L. Bagley and P.J. Torvik, On the fractional calculus model of viscoelastic behavior, Journal of Rheology 30(1) (1986), 133-155.

[9] K.-J. Bathe, Finite element procedures in engineering analysis, Prentince-Hall, Englenwood Cliffs, 1982.

[10] M. Caputo, Vibrations of an infinitive viscoelastic layer with a dissipative memory, Journal of Acoustical Society of America 56(3) (1974), 897-904.

[11] M. Caputo and F. Mainardi, A new dissipation model based on memory mechanism, Pure and Applied Geophysics 91(8) (1971), $134-147$.

[12] M. Caputo and F. Mainardi, Linear models of dissipation in anelastic solids, Rivista del Nuovo Cimento 1(2) (1971), 161-198.

[13] M. Enelund and P. Olsson, Damping described by fading memory analysis and application to fractional derivative models, International Journal of Solids and Structures 36 (1999), 939-970.

[14] J. Freundlich and M. Pietrzakowski, FEM simulation of the vibration girder measurement using piezoelectric sensors, Machine Dynamics Research 34 (2010), 28-36.

[15] J.F. Hall, Problems encountered from the use (or misuse) of Rayleigh damping, Earthquake Engineering And Structural 35 (2006), 525545.

[16] K. Hedrih (Stevanovic), The transversal creeping vibrations of a fractional derivative order constitutive relation of nonhomogeneous beam, Mathematical Problems in Engineering (2006), 1-18.

[17] J.T. Machado, V. Kiryakova and F. Mainardi, Recent history of fractional calculus, Communication in Nonlinear Science and Numerical Simulatation 16 (2011), 1140-1153.

[18] F. Mainardi, Fractional calculus and waves in linear viscoelastisity: An introduction to mathematical models, London, Imperial College Press, 2009.

[19] A.D. Nashif, D.I.G. Jones and J.P. Henderson, Vibration damping, John Wiley \& Sons, New York, 1985.

[20] I. Podlubny, Fractional differential equations, San Diego, Academic Press, 1999.

[21] Y.A. Rossikhin and M.V. Shitikova, New approach for the analysis of damped vibrations of fractional oscillators, Shock and Vibration 16 (2009), 365-387.

[22] Y.A. Rossikhin and M.V. Shitikova, Application of fractional calculus for dynamic problems of solid mechanics: Novel trends and recent results, Applied Mechanics Reviews 63 (2010), 1-51.

[23] J.F. Semblat, Rheological interpretation of rayleigh damping, Journal of Sound and Vibration 206(5) (1997), $741-744$.

[24] L.E. Suarez and A. Shokooh, An eigenvector expansion method for the solution of motion containing fractional derivatives, Transaction of the ASME Journal of Applied Mechanics 64 (1997), 629-635.

[25] C.W de Silva, Vibration: Fundamentals and practice, Boca Raton, CRC Press, 2000. 

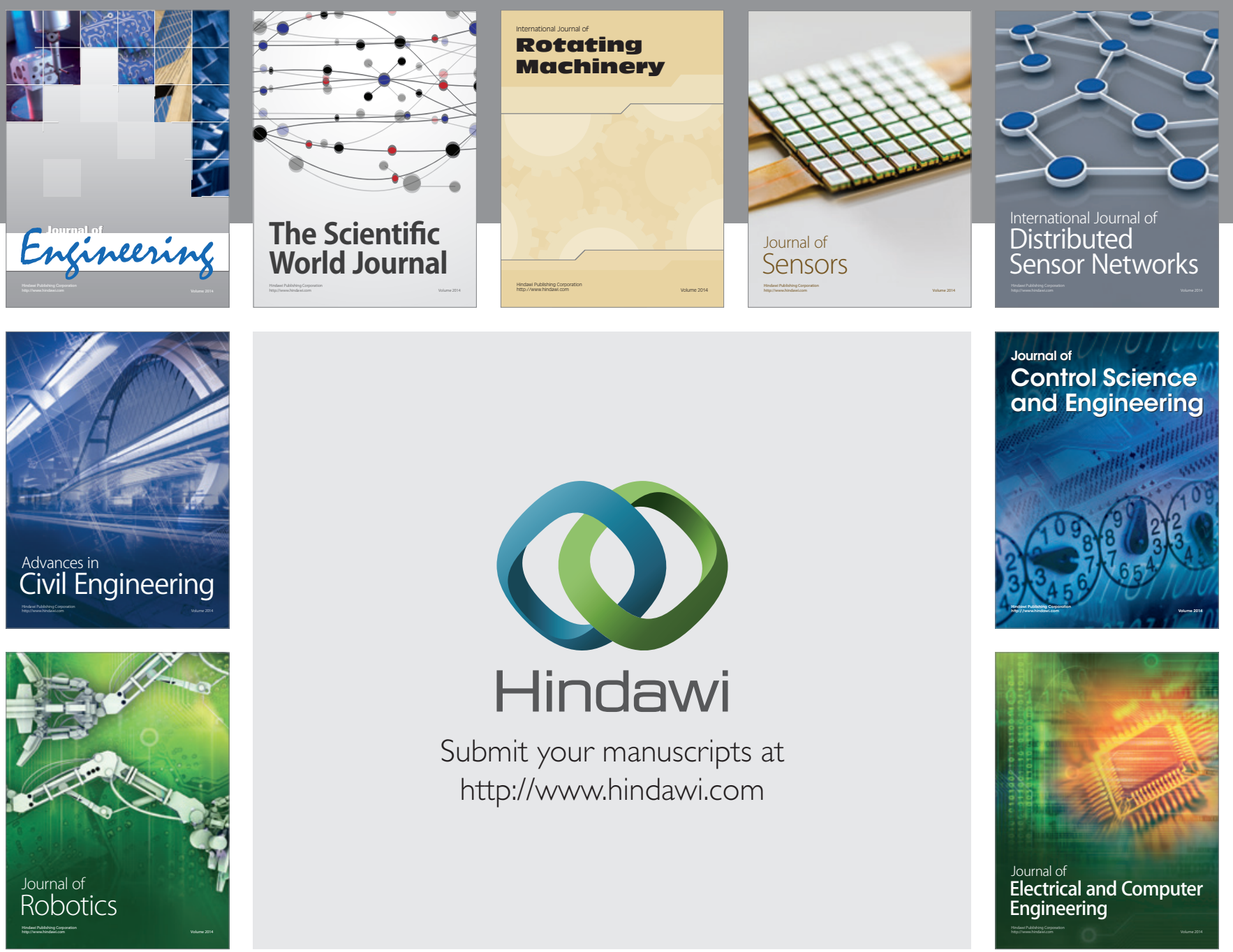

Submit your manuscripts at

http://www.hindawi.com
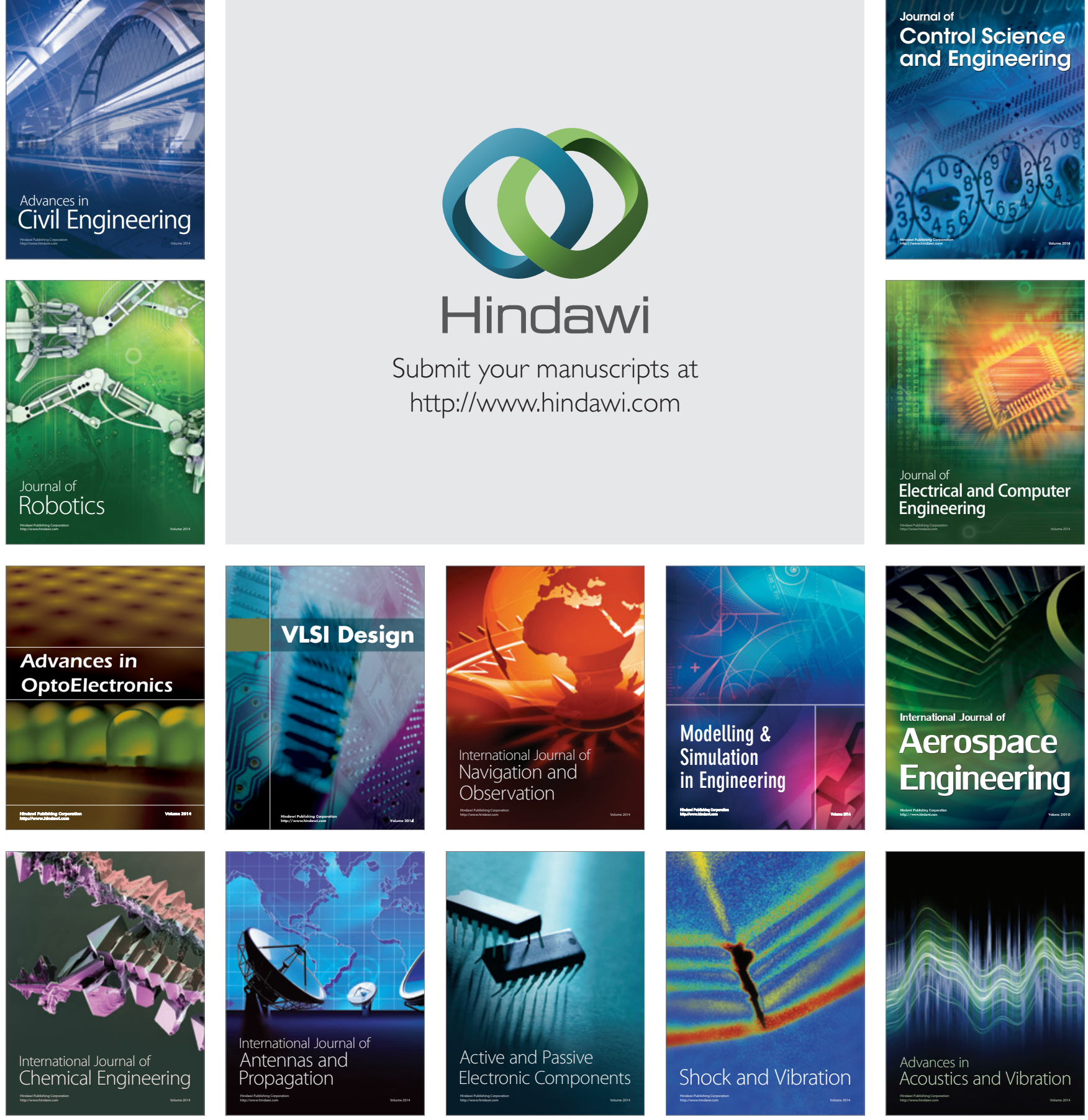